\title{
Serial extraction: 20 years of follow-up
}

Renato Rodrigues de ALMEIDA¹, Marcio Rodrigues de ALMEIDA¹, Paula Vanessa Pedron OLTRAMARI-NAVARRO'1, Ana Cláudia de Castro Ferreira CONTI ${ }^{1}$, Ricardo de Lima NAVARRO ${ }^{2}$, Karen Regina Siqueira de SOUZA ${ }^{3}$

\footnotetext{
1- DDS, MSc, PhD, Professor, Department of Orthodontics, University of North Paraná - UNOPAR, Londrina, PR, Brazil.

2- DDS, MSc, PhD, Oral and Maxilofacial Surgeon, Professor, Department of Orthodontics, University of North Paraná - UNOPAR, Londrina, PR, Brazil.

3- DDS, MSc, Former Graduate student, Department of Orthodontics, University of North Paraná - UNOPAR, Londrina, PR, Brazil.
}

Corresponding address: Paula Vanessa Pedron Oltramari-Navarro - Rua Paranaguá, 803. Apto 92 - 86020-030 - Londrina - PR - Phone: (43) 9134-4499 -e-mail: pvoltramari@hotmail.com

Received: April 5, 2010 - Modification: May 30, 2010 - Accepted: October 26, 2010

\section{ABSTRACT}

\begin{abstract}
$T^{n}$ is paper reports a case treated by a serial extraction program at the mixed dentition stage followed by a corrective orthodontic treatment, with a long-term follow-up period. Twenty years after the interceptive treatment, a harmonious face was observed along with treatment stability in the anterior posterior direction, deep overbite (which has been mentioned as a disadvantage of the serial extraction program), and a small relapse of anterior tooth crowding. All these conditions have been regarded as normal occurrences for most orthodontic treatments with a long-term follow-up period. This case report demonstrated that the establishment of a serial extraction protocol determined relevant esthetic changes that afforded an improvement of the patient's self-esteem, with a positive social impact. Furthermore, the low cost of this protocol permits the use of this therapy with underprivileged populations. It is important to emphasize that an early correction of tooth crowding by this protocol does not guarantee stability, but small relapses do not invalidate its accomplishment.
\end{abstract}

Key-words: Interceptive orthodontics. Serial extraction. Crowding. Stability.

\section{INTRODUCTION}

The practice of serial dental extractions aims at correcting definitive primary crowding, of a genetic etiology ${ }^{17}$. The correction of the real discrepancy between the tooth mass and the osseous base is performed by specific extractions as from the beginning of the mixed dentition, following a predetermined strategic sequence that allows the spontaneous alignment of the permanent teeth ${ }^{6}$. The best stage for diagnosing definitive primary crowding, and for initiating the serial extraction protocol is, undoubtedly, the stage when the permanent incisors erupt into the oral cavity $5,7,8,16,19$. This protocol is indicated for Class I malocclusions with negative tooth/bone discrepancy, or when there is no need for dental arch expansion, or still, should the expansion be necessary, when it is not sufficient to result in a tooth size/arch length compatibility 6,14.

A serial extraction protocol involves two distinct stages, demanding thus a long-term period of observation ${ }^{4}$. The extraction sequence of the first stage comprises the removal of the primary lateral incisors, thereby affording an immediate alignment of the permanent central incisors; and that of primary canine teeth, thereby permitting the alignment of the already crowded permanent lateral incisors. Thus, the space deficit problem is solved in this first stage, by only eliminating the primary teeth. The second stage of the program occurs during the second transitional period of the mixed dentition, when the extraction of the primary molars and first premolars ${ }^{4}$ is carried out (Figure 1). Few studies have been found in literature reporting on a long follow-up period ${ }^{1,11,12}$. Therefore, the aim of this paper is to report a case treated by a serial extraction program at the mixed dentition stage followed by a corrective orthodontic treatment, with 20 years of follow-up. This record will demonstrate that the establishment of a serial extraction protocol determines relevant functional and aesthetic changes, with a low-cost therapy. Additionally, the majority of the results obtained 
at the end of the treatment could be maintained, despite a small relapse of the overbite and tooth crowding, which are expected after long periods in all orthodontic treatments.

\section{CASE REPORT}

An eight-year-and-two-month-old Caucasian female patient had a chief complaint of an upper inter-incisor diastema. In the facial analysis, the patient showed a harmonious face and a normal

\begin{tabular}{|l|}
\hline \multicolumn{1}{|c|}{ Serial extraction $-\mathbf{1}^{\text {st }}$ stage } \\
\hline Dental extractions: $52,62,72,82,53,63,73,83$ \\
\hline Desirable period: first transitional period of the mixed dentition \\
\hline Goal: \\
\hline Extraction of the primary lateral incisors and canine teeth to promote a permanent incisor alignment- \\
\hline Begin on the lower arch \\
\hline \multicolumn{1}{|c|}{ Serial extraction $-\mathbf{2}^{\text {nd }}$ stage } \\
\hline Dental extractions: $54,64,74,84,55,65,75,85,14,24,34,44$ \\
\hline Desirable period: second transitional period of mixed dentition \\
\hline Goal: \\
\hline Extraction of the primary molars to accelerate first bicuspid eruption \\
\hline Extraction of first bicuspids to achieve permanent canine teeth distalization and eruption \\
\hline Begin on the lower arch \\
\hline
\end{tabular}

Figure 1- Operative phases of the serial extraction program

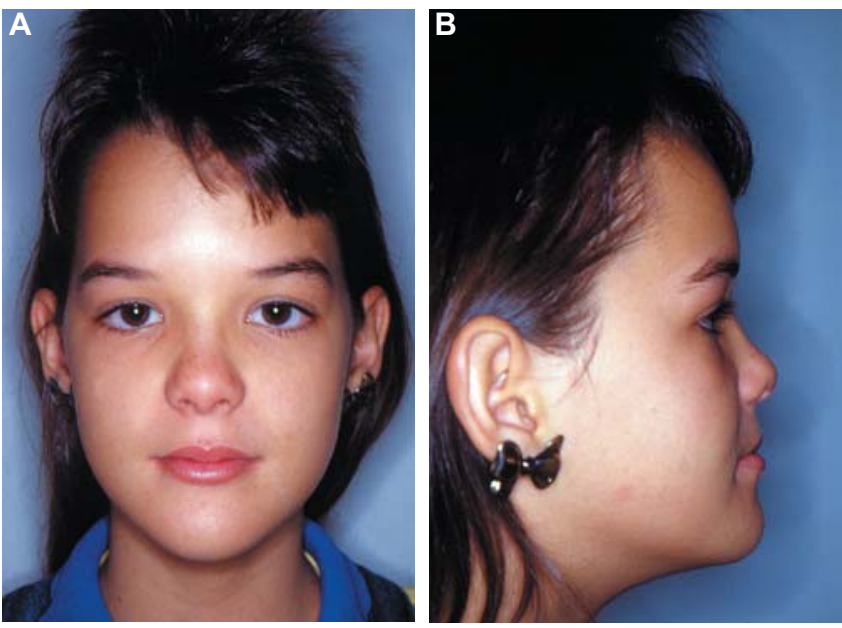

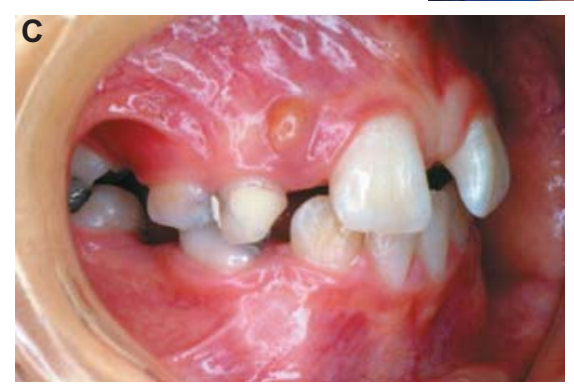

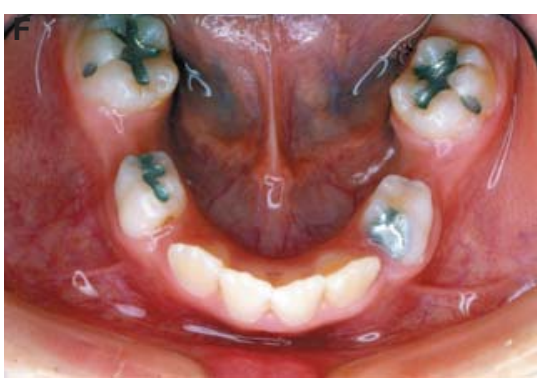

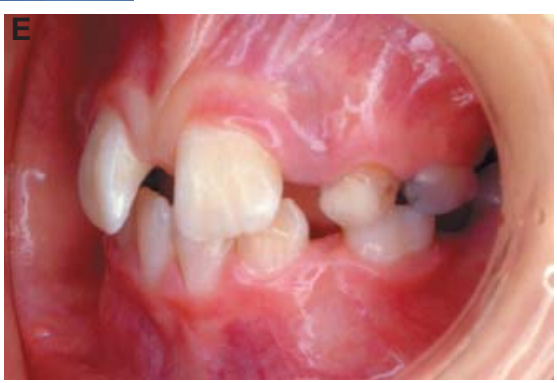

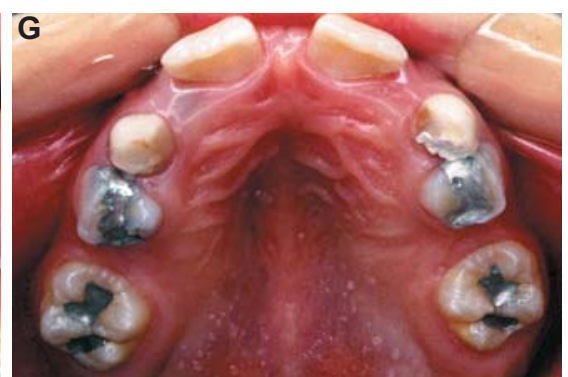

Figure 2- Pretreatment extraoral (A and B) and intraoral $(C-G)$ photographs (the parents authorized the publication of these pictures) 
labial posture. In the intraoral clinical examination, the presence of a large diastema was observed, together with several early extractions of primary

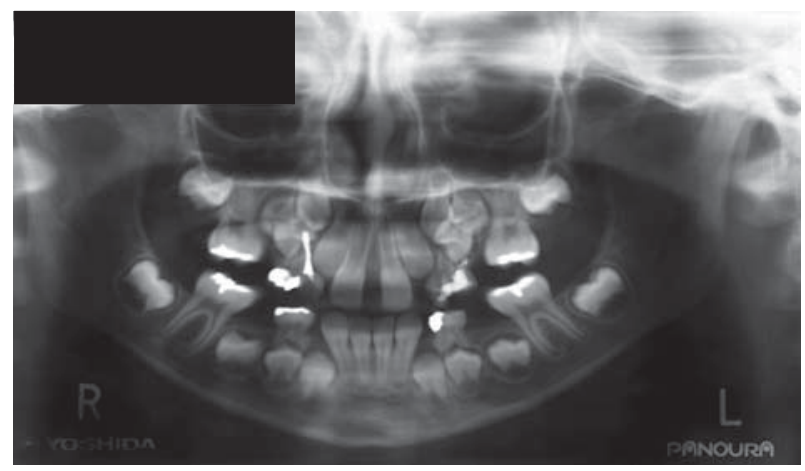

Figure 3- Initial panoramic radiograph
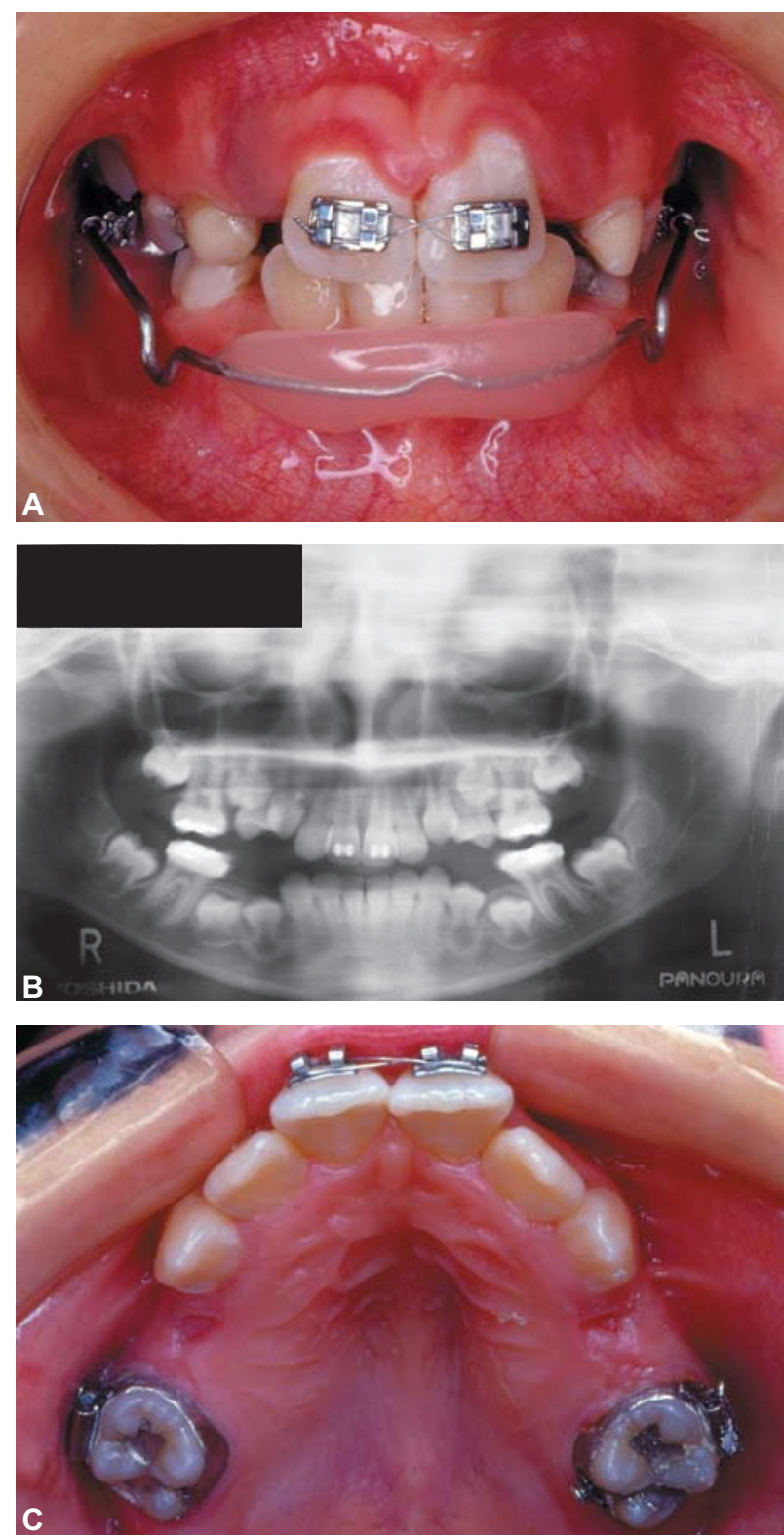

Figure 4- Intermediate phase showing the anchorage preparation in the upper arch, and the reduction of the diastema $(\mathrm{A}-\mathrm{C})$ teeth, with great space loss, and, consequently, a reduction of upper and lower arch length (Figure 2).

\section{Radiographic exam}

In the panoramic radiograph, presence of multiple extractions of primary teeth was verified, with consequent lack of space due to retroclination of the permanent teeth, as was the presence of all permanent teeth, which had either erupted or were at several developmental stages, except for the third molars (Figure 3 ).

\section{Treatment}

The treatment protocol was composed of two stages: one interceptive and one comprehensive. In the interceptive phase, a serial extraction program was performed, which aimed especially at correcting the discrepancies of the dental arch, fostering favorable conditions for a normal development of the occlusion. Due to the great lack of space, initial planning comprised anchorage preparation: a) an extraoral appliance in the upper arch (cervical headgear) to obtain upper molar distalization; b) an active lip bumper, on the lower arch; c) bracket installation on the upper incisors to close the diastema, and to provide space for lateral incisor eruption. Still in this stage, primary first molar extractions were indicated to accelerate eruption of first premolars, which were to be extracted sequentially to create space for the permanent canine teeth (Figure 4).

After the eruption of all permanent teeth, at the end of interceptive phase, new radiographs were obtained to plan the comprehensive phase (Figure 5). In this phase, a fixed appliance was installed aiming to align, level, and correct the axial inclinations, and small diastemas (Figure 6 ). Figure 7 shows the treatment finalization, when the patient was oriented regarding the use of retainers: an upper Hawley appliance, and a lower $3 \times 3$ retainer.

Twenty years after the end of the interceptive treatment, a new follow-up appointment was scheduled. It could be noted that the facial proportion balance was preserved, with only a small relapse at the upper arch, and lower anterior crowding (Figure 8).

\section{DISCUSSION}

The accomplishment of a serial extraction protocol affords early correction of tooth size/ arch length discrepancy, improvement of selfesteem, better oral hygiene, a better chewing function, and a reduction of the period of corrective orthodontic treatment ${ }^{3,19}$. Despite the positive effects demonstrated by this treatment protocol, 

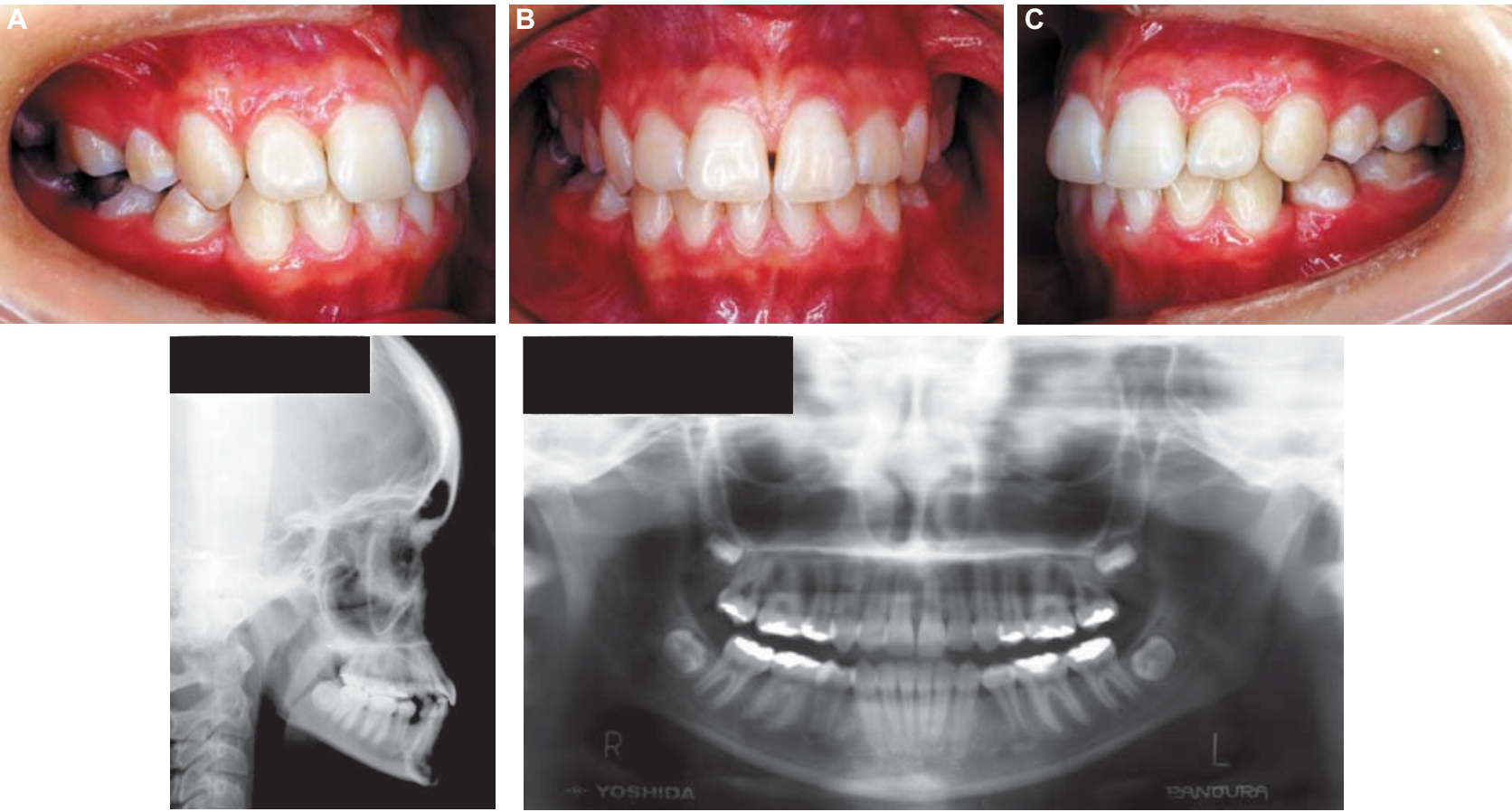

Figure 5- Intraoral photographs (A-C), lateral cephalogram (D), and panoramic radiograph (E) at the end of the interceptive stage
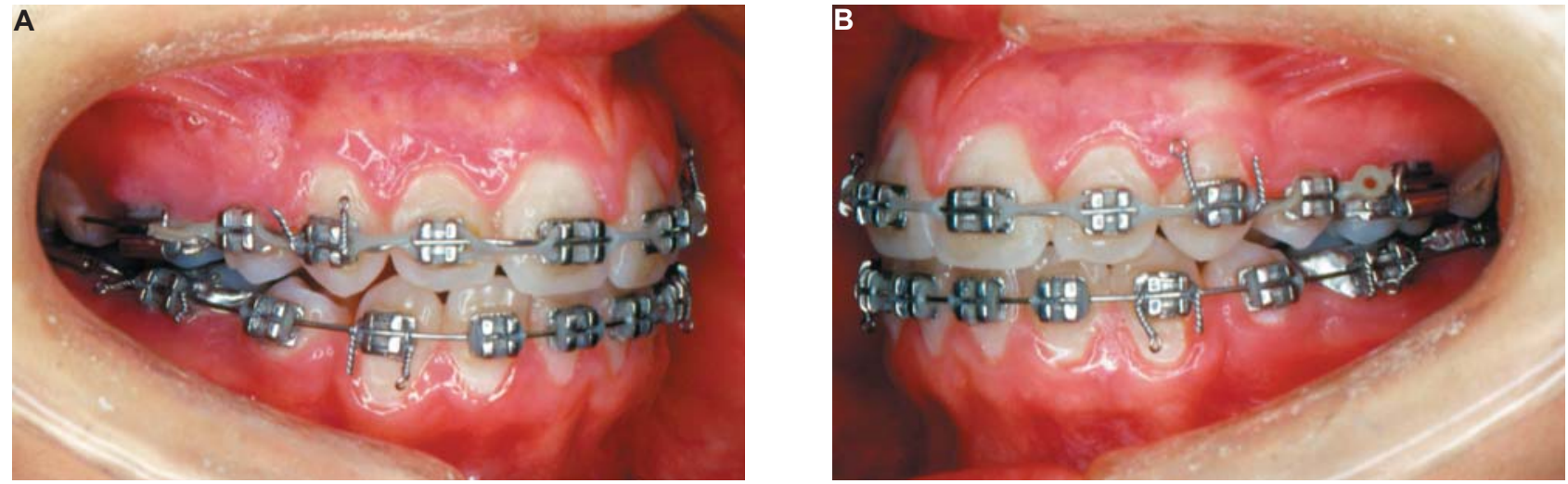

Figure 6- Intraoral photographs showing the fixed appliance (A and B)

some disadvantages may be pointed out, such as the need of specialized long-term follow-up, possible remaining spaces in the area of the extracted teeth, retroclination of the incisors, overbite, increase of both the Spee curve and the profile concavity, possibility of tongue interposition, and periodontal problems.

In spite of the advantages and disadvantages of the serial extraction procedure, there have been controversies on the best intervention in cases of tooth/bone discrepancy, in that a few questions remain: whether to increase arch length; to reduce the tooth mass; or to wait for the exfoliation of the primary second molars, with interception only if there is an early loss of primary canine teeth ${ }^{1,14}$. All the aforementioned possibilities have been described in the literature ${ }^{9,12}$. An increase of arch width (expansion) did not show stability when treating the negative tooth/bone discrepancy, at mixed dentition, in that $89 \%$ of the patients had unsatisfactory results, with an irregularity index of $6.06 \mathrm{~mm}$ after a 7.5-year follow-up ${ }^{12}$. On the other hand, the studies performed with premolar extractions after a 10-year follow-up showed an irregularity index of $4.39 \mathrm{~mm}^{11}, 3.50 \mathrm{~mm}^{13}$, and $3.09 \mathrm{~mm}^{9}$, respectively.

Concerning the long-term stability of serial extraction, it has stated that the results do not differ from those of conventional orthodontic treatments during permanent dentition ${ }^{10,15}$. For a long time, researchers thought that the relapse, in all cases treated, was part of an expected final result. Riedel ${ }^{15}$ (1960) claimed that teeth that were moved orthodontically had a tendency to return to their initial positions. This author also stated that corrections done during growth periods showed minimal relapse. Van Der Linden and Boersma ${ }^{18}$ (1987) corroborated these results, and stated that 

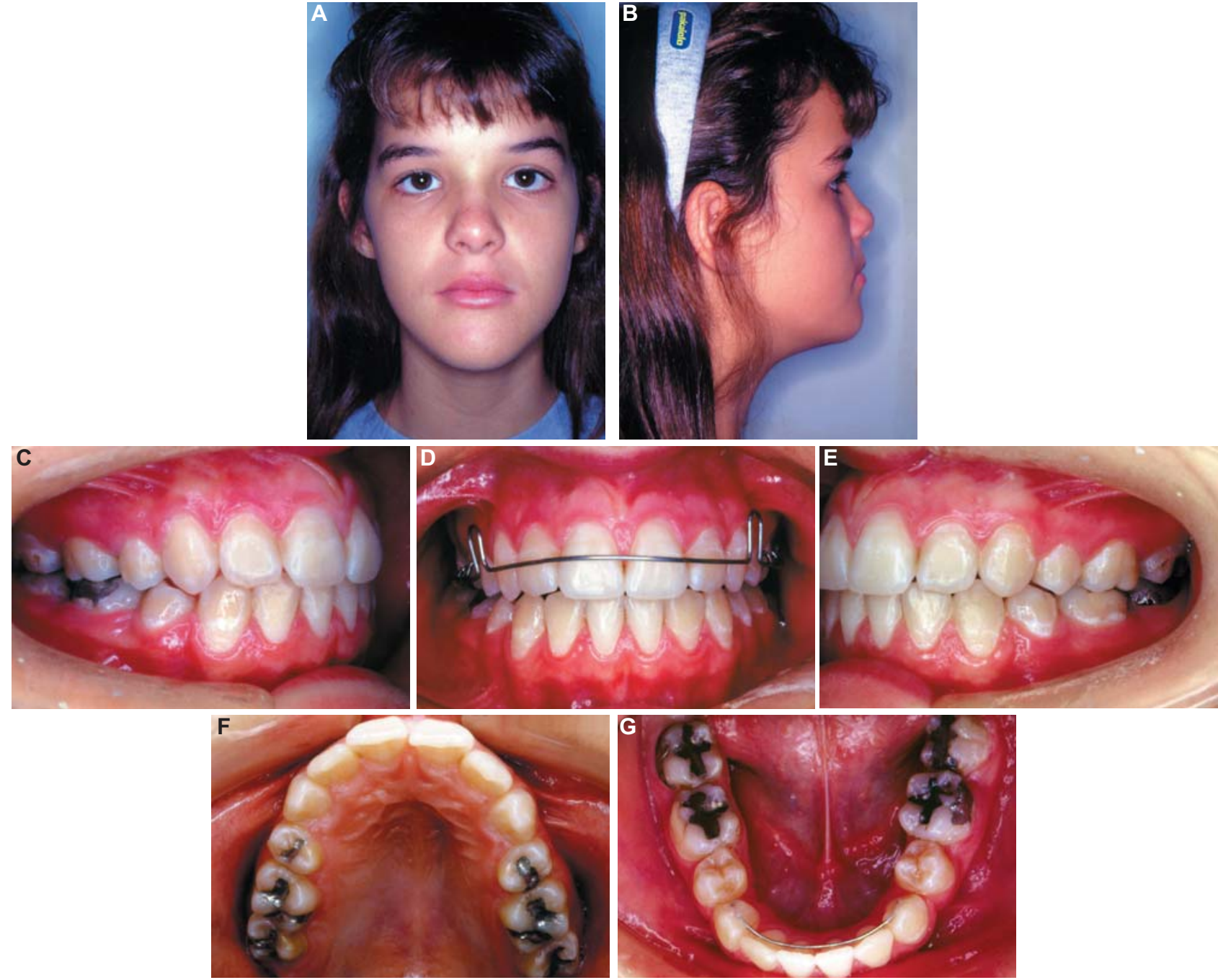

Figure 7- End of comprehensive phase: extraoral $(A$ and $B$ ) and intraoral $(C-G)$ photographs (the parents authorized the publication of these pictures)

the completion of an orthodontic treatment with satisfactory intercuspation, proximal contacts, and buccal-lingual inclination would diminish the chance of relapse.

This paper aimed to demonstrate a serial extraction protocol through the presentation of a clinical case. A degree of relapse, compatible with that observed in orthodontic posttreatment evaluations, could be noted in this patient. It is important to emphasize the lack of long-term follow-up studies that demonstrate the changes occurred after this procedure. Few reports followed up on patients as to the effects of a serial extraction ${ }^{1,11,12}$ for more than 10 years. The present study reported on a 20-year follow-up after serial extraction. The results presented here reinforce the viability of this treatment protocol, since there was a considerable esthetic improvement, at an early developmental stage ${ }^{2}$. However, despite the good outcomes achieved in the present case, further long-term clinical investigations are necessary to assure the stability of a serial extraction protocol.

\section{CONCLUSION}

The long-term follow-up of the clinical case reported in this paper demonstrates that undertaking a serial extraction protocol determined relevant esthetic changes that afforded an improvement of the patient's self-esteem, resulting in a positive social impact. Furthermore, the low cost of this protocol permits the use of this therapy in underprivileged communities. It is important to emphasize, though, that the early correction of tooth crowding by this protocol does not guarantee stability, but small relapses do not invalidate its accomplishment. 

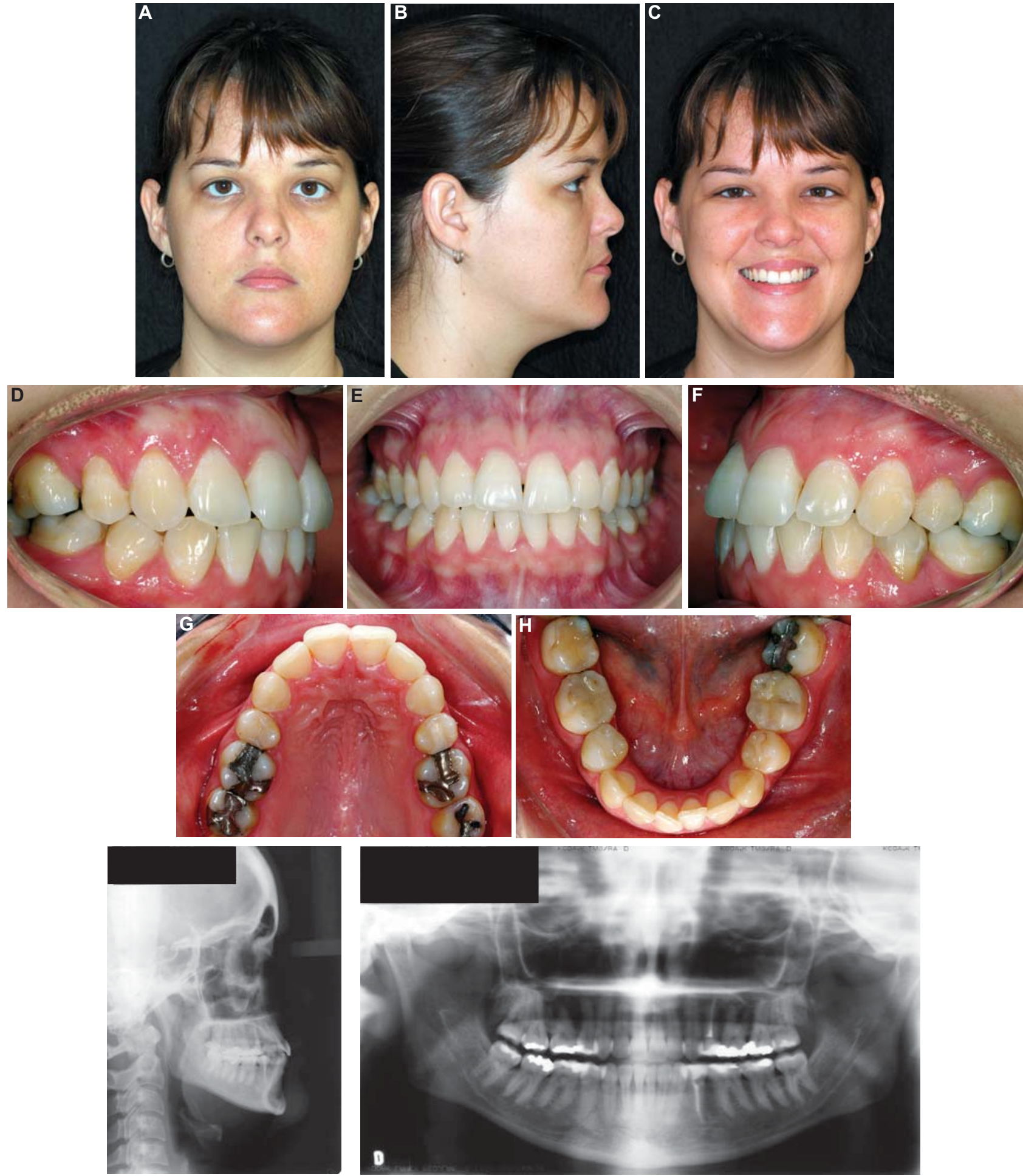

Figure 8- Twenty-year follow-up after the interceptive stage: extraoral $(A-C)$ and intraoral $(D-H)$ photographs, lateral cephalogram (I), panoramic radiograph $(\mathrm{J})$ (the parents authorized the publication of these pictures)

\section{REFERENCES}

1- Boley JC. Serial extraction revisited: 30 years in retrospect. Am J Orthod Dentofacial Orthop. 2002;121(6):575-7.

2- Carter GA, McNamara JA Jr. Longitudinal dental arch changes in adults. Am J Orthod Dentofacial Orthop. 1998;114(1):88-99. 3- Dale JG. Serial extraction... nobody does that anymore! Am J Orthod Dentofacial Orthop. 2000;117(5):564-6.

4- Dale JG, Brandt S. Dr. Jack G. Dale on serial extraction. 3. J Clin Orthod. 1976;10(3):196-217.
5- Dewel BF. Prerequisites in serial extraction. Am J Orthod. 1969;55:533-9.

6- Dewel BF. Serial extraction in orthodontics: indications, objectives, and treatment procedures. Am J Orthod. 1954;40:90626.

7- Dewel BF. Serial extraction, second premolars, and diagnostic precautions. Am J Orthod. 1978;73(5):575-7.

8- Graber TM. Serial extraction: a continuous diagnostic and decisional process. Am J Orthod. 1971;60(6):541-75. 
9- Haruki T, Little RM. Early versus late treatment of crowded first premolar extraction cases: postretention evaluation of stability and relapse. Angle Orthod. 1998;68(1):61-8.

10- Little RM. Stability and relapse: early treatment of arch length deficiency. Am J Orthod Dentofacial Orthop. 2002;121(6):57881.

11- Little RM, Riedel RA, Engst ED. Serial extraction of first premolars - postretention evaluation of stability and relapse. Angle Orthod. 1990;60(4):255-62.

12- Little RM, Riedel RA, Stein A. Mandibular arch length increase during the mixed dentition: postretention evaluation of stability and relapse. Am J Orthod Dentofacial Orthop. 1990;97(5):393404.

13- McReynolds DC, Little RM. Mandibular second premolar extraction - postretention evaluation of stability and relapse. Angle Orthod. 1991;61(2):133-44.
14- Proffit WR. The timing of early treatment: an overview. Am J Orthod Dentofacial Orthop. 2006;129(4 Suppl):S47-9.

15- Riedel RA. A review of the retention problem. Angle Orthod. 1960;30:179-99.

16- Ringenberg Q. Serial extraction: stop, look and be certain. Am J Orthod. 1964;50:327-36.

17- Van Der Linden F. Theoretical and practical aspects of crowding in the human dentition. J Am Dent Assoc. 1974;89:13953.

18- Van Der Linden F, Boersma H. Indications and contraindications for treatment. In: Diagnosis and treatment planning in dentofacial orthopedics. Chicago: Quintessence; 1987. p. 165-83. 19- Yoshihara T, Matsumoto Y, Suzuki J, Sato N, Oguchi H. Effect of serial extraction alone on crowding: relationships between tooth width, arch length, and crowding. Am J Orthod Dentofacial Orthop. 1999;116(6):691-6. 